\title{
Do researchers know what they are doing?
}

\section{PD Singleton}

Research governance has a number of functions: the first is to protect the interests of the research participants themselves and to ensure that they understand the implications of and agree to the risks involved in the particular research project; second, there is the assurance of the research methodology, both in its scientific effectiveness and in its limitation of risks to the individuals concerned; finally, there is a cost-effectiveness or opportunity-cost review to ensure that this piece of research should proceed rather than another, or even not at all.

Generally, the first is the domain of the research ethics committees (RECs), whereas the last is down to funders and/or the healthcare system where it is involved, although RECs might decide that the risks to participants are not worth the likely scientific benefits. The second area is often a bone of contention where researchers may feel it is not for RECs to adjudge their expertise in deciding on a scientific methodology, and funders may not be happy with the increased costs that RECs may effectively add to a project through additional consent procedures.

The article by Knowles et $a l^{1}$ in this issue nicely illustrates some of the threeway tension between researchers who are focused on their scientific interests and want to just do it, patients who may have strong confidentiality and privacy interests to protect and regulatory bodies who want to be seen to be providing that protection.

The article also raises the question of whether researchers know enough about the workings of RECs or the wider approval processes or whether RECs know enough about the wider scope of research rather than clinical trials where the questions of more immediate harm to participants are paramount.

Researchers spend a lot of time and energy developing a research protocol, pulling together a team and getting

Correspondence to Peter Desmond Singleton, Centre for Health Informatics and Multiprofessional Education, University College London, Holborn Union Building, Highgate Hill, London N19 5LW, UK; peter.singleton@chi-group.com funding for the project. It can then be very frustrating to go before an REC and have your proposal rejected because of ethics issues, when all you are trying to do is save the world! However, many researchers simply have not had the support or training on how to prepare for an ethics review and to make sure that their project properly supports patients' interests.

\section{Perspective on the paper by Knowles et al (see page 14)}

This leads to one of the main ethics requirements: to provide patients with a choice about whether to be involved or not. Clearly, where some intervention is involved (whether clinical or simply a questionnaire or interview), patients can choose not to participate. Even if it is only their data being used, providing them with a choice is rarely great hardship, is evidently fair and, perhaps more importantly, engenders and supports the ongoing trust that most of the public have in research. ${ }^{2} 3$

It is important to remember that patients are not just statistical objects to be counted and measured. For some of them, their medical data are sensitive and could cause significant embarrassment or loss if accidentally or deliberately revealed; some simply have strong feelings about their privacy even if no obvious harm were to befall them.

Research applications have a tendency to assume that scientific accuracy trumps patient choice, often claiming that any patient opt-out would invalidate the research, often without realising the implication that the study itself must be of questionable usefulness if its likely conclusions are so uncertain. It is important for researchers to understand that from a REC perspective, the reverse is the case: patient choice trumps scientific need.

Researchers need to follow the UK NHS Confidentiality Code of Practice ${ }^{4}$ in providing choice where practicable.
Focusing on choice rather than consent, which is more of a lawyer's concept and takes one away from what the person themselves may want, would greatly help the design of any research protocol.

Involving some patient representatives in the study design helps keep things grounded in reality and wins brownie points with most approvals bodies. In one study, participants were asked to sign five different consent forms, including one to say they had not changed their minds since signing the other four. Having a lay response that this was plain silly would, perhaps, have helped persuade the REC involved to take a more patient-centric and reasonable approach to providing choice rather than enforcing consent.

This illustrates another tension, the variability between RECs. One project with which I was involved made the mistake of approaching an REC with little experience of large-scale medical databases, either as applications or of the issues involved. With the benefit of hindsight, we should have applied to one that had already dealt with one or more similar projects, and so they would have been better prepared to consider our protocols in a more measured fashion. Nevertheless, their scrutiny did help us improve our proposed approach, and although it limited what we could do, it probably had little net effect on the scientific output of our project. Fortunately, REC review is now optional for such studies, but this still shows the need for some experience of the system and the variability of interpretation. The virtue of independent scrutiny by RECs has the necessary consequence of possible inconsistency in decisions, though one would hope not as a consequence of inconsistency of skills or knowledge.

RECs are often geared up to consider clinical trials, where risks to the individuals are potentially high, but costs of the consent process are relatively low, rather than epidemiological studies, particularly with large number of individuals, where the costs of the consent process will be a significant proportion of the likely project costs and the reduction in risks to the individuals concerned minimal (see Singleton and Wadsworth ${ }^{5}$ for a more detailed consideration of this point). Too often, a clinical trial mindset is applied without consideration of the different risks and issues for a different type of research project. 
It is important also to realise that delays can also occur in the publication of papers and the ethics approval process, so that there have been a number of changes in the landscape since the project started and the paper was first drafted.

In the UK, for example, there has been much effort in recent years to coordinate forms between approval bodies, to only ask for the information once and to provide quicker decision making and faster responses to applicants. A number of research studies now clearly do not have to have REC approval or can seek it optionally. See http://www.nres. npsa.nhs.uk for more details about the Integrated Research Application System available at https://www.myresearchproject.org.uk/Signin.aspx.

There is the broader question of the role of research in medicine and healthcare delivery. We currently treat research as an optional exercise, an academic pursuit that is irrelevant to the public at large. Surely nothing could be further from the truth: it is research that helps us understand what works and what does not; it is one of the key mechanisms by which the health service improves. The UK body, the National Institute for Health and Clinical Excellence, relies on research results to guide its decisions; practitioners pay heed to the results, though perhaps less than we would like to, hence the huge variations in care delivery. We should make research a key plank of the quality agenda and recognise it for what it is, an essential part of medicine because it needs to be practised.

It would seem to me to be better that we effected a social contract with the public that presumes that they would want their medical records (suitably protected and supervised, of course) used to improve the quality of their health and healthcare. They could still choose to have no part of this if they wanted, in the same way that we allow people to refuse treatment, if they have objections to the principle or the practice of research. This would allow a far better use of resources for all and a solution to the consent-toconsent problem, the only downside of which would be fewer papers such as this.

I would note that some organisations make a point of informing patients routinely about the use of medical records, particularly for research, and so are already leading the way to establishing such an understanding with and for the public.

It is clear that researchers need to know more about the approval processes before they start, including how it is changing, and the same is true for the approvals bodies themselves, which need to be more aware of how research is also changing, particularly in the areas of data mining to the benefit of all. In the UK, the Medical Research Council has produced a very useful Data and Tissues Toolkit at http://www.dt-toolkit.ac.uk, which helps guide researchers through the approvals maze. Perhaps, we need a similar guide to research projects for RECs.

\section{Competing interests None.}

Provenance and peer review Commissioned; internally peer reviewed.

Accepted 21 April 2010

Published Online First 16 June 2010

Arch Dis Child 2011;96:3-4.

doi:10.1136/adc.2009.181636

\section{REFERENCES}

1. Knowles RL, Bull C, Wren C, et al. Ethics, governance and consent in the UK: implications for research into the longer-term outcomes of congenital heart defects. Arch Dis Child 2011; 96:14-20.

2. Ipsos MORI. The use of personal health information in medical research. Medical Research Council, 2007.

3. Shepherd R, Barnett J, Cooper H, et al. Public attitudes to research governance: a qualitative study in a deliberative context. London: Wellcome Trust, 2007.

4. UK Department of Health. NHS confidentiality code of practice, Nov 2003.

5. Singleton $\mathbf{P}$, Wadsworth $\mathbf{M}$. Consent for the use of personal medical data in research. BMJ 2006;333:255-8. 